\title{
Peripheral facial paralysis as a consequence of acute otitis media: Physiopathology, associated factors and treatment
}

\author{
Daniel NA Teixeira, Matheus V de Figueiredo, Rodolfo G Fernandes, Eder B Muranaka, José AA Lobo, José F de Sales Chagas* and José E \\ Baptistella
}

Department of Otorhinolaryngology-Head and Neck Surgery, Prefeito Edivaldo Orsi Hospital Complex, Rede Mário Gatti, Campinas, Brazil

\section{Introduction}

Acute otitis media (AOM) is a common disease in clinical practice. Facial nerve palsy is an uncommon complication of AOM, with an incidence of $0.005 \%$. It is estimated that this incidence was 0.5 to $0.7 \%$ before the emergence of antibiotic therapy. This complication is believed to be due to inflammatory edema in the facial nerve canal with consequent ischemia and neuropraxia [1]. The exact pathophysiological mechanism by which acute otitis media leads to facial paralysis is unclear. It is assumed that there is a relationship with the anatomical intimacy between the facial nerve and the temporal bone $[2,3]$.

In this study, we report a case of facial paralysis in a child with acute otitis media. We also discuss pathophysiological mechanisms, associated factors, prognosis and recommended treatments.

\section{Case report}

A 12-year-old-girl presented with a 1-day history of facial paralysis on the right side. Two days earlier, she had otalgia and paraesthesia in the right hemiface, associated with ear fullness and objective vertigo.

Physical examination showed irritability and fever to $101.3^{\circ} \mathrm{F}$. She also had facial paralysis House-Brackmann grade III [4], that is, obvious but not disfiguring difference between two sides, noticeable but not severe synkinesis, complete closure of the eye with effort, mouth asymmetry with maximum effort (Figure 1). Otoscopy showed a bulging, hyperemic right tympanic membrane and the presence of retrotympanic fluid. Left otoscopy showed only a hyperemic tympanic membrane. There were no vesicles suggestive of Herpes Zoster.

Pure tone audiometry (PTA) showed a right conductive hearing loss (air-bone gap of $15 \mathrm{~dB}$ ). Computed tomography (CT) scan showed no signs of facial nerve canal dehiscence, it showed only partial opacification of the mastoid air cells (Figure 2).

Treatment started with amoxicillin/clavulanate 500/125 mg PO q8hr for 10 days, prednisone $20 \mathrm{mg}$ PO daily for 7 days. Myringotomy was performed on the right ear with drainage of purulent secretion. On the 3rd day of hospitalization, an electroneuromyography was performed, which showed facial nerve degeneration of $60 \%$. On the 10th day of treatment, there was partial regression of facial paralysis with grade II on the House-Brackmann scale, that is, slight weakness noticeable on close inspection, complete closure of the eye with minimum effort, slight asymmetry of the mouth. The patient also reported otalgia improvement and absence of fever. On the 20th day of follow-up, the patient had normal facial movements, in addition to total otalgia regression.

\section{Discussion}

Facial paralysis can be congenital, traumatic, infectious, neoplastic or idiopathic (Bell's palsy), which is the most common cause today. In the past, the most common cause of acute facial nerve palsy in children was AOM. After the emergence of broad-spectrum antibiotic therapy, the clinical course of acute otitis media was changed, and coalescent mastoiditis became less common [5]. Currently, facial paralysis is a rare complication of AOM, with an incidence of $0.005 \%$. It is estimated that this incidence was 0.5 to $0.7 \%$ before the emergence of antibiotic therapy [5]. The term otomastoiditis is used when mastoiditis and acute otitis media occur concurrently. This causes blockage of the normal aeration of the mastoid cells, which reduces the vascular perfusion of the mucosa and decreases the tissue penetration of antibiotics. The inflammatory process on the facial nerve canal, through canal dehiscence or invasion of infectious microorganisms, results in inflammation and edema of the nerve inside its canal. The venous return decreases and the pressure increases on the nerve, which leads to nerve dysfunction ${ }^{7}$. As the limits of the facial nerve canal are narrow, the accumulation of purulent secretion

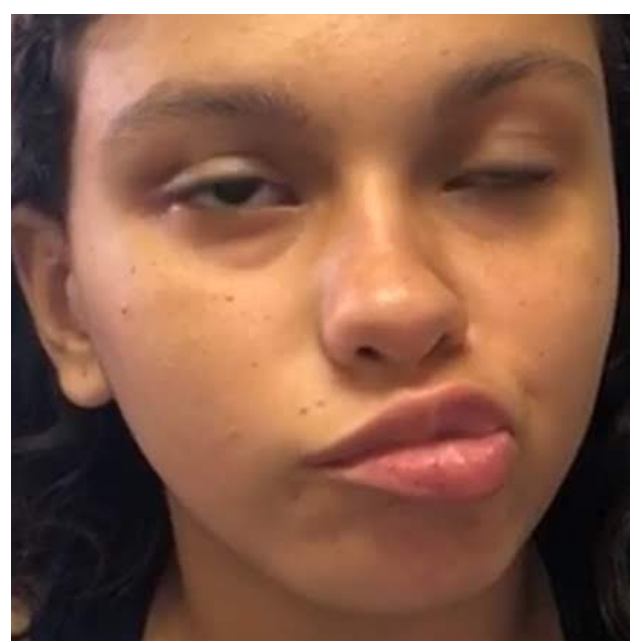

Figure 1. Facial paralysis on the right, with mouth asymmetry and difficulty in closing the eyelids

${ }^{\star}$ Correspondence to: José Francisco de Sales Chagas, Department of Otorhinolaryngology-Head and Neck Surgery, Prefeito Edivaldo Orsi Hospital Complex, Rede Mário Gatti, Campinas, Brazil, Tel: +55 19 99765-3010; E-mail: josechagas@ig.com.br

Received: January 03, 2020; Accepted: January 20, 2020; Published: January 24 2020 


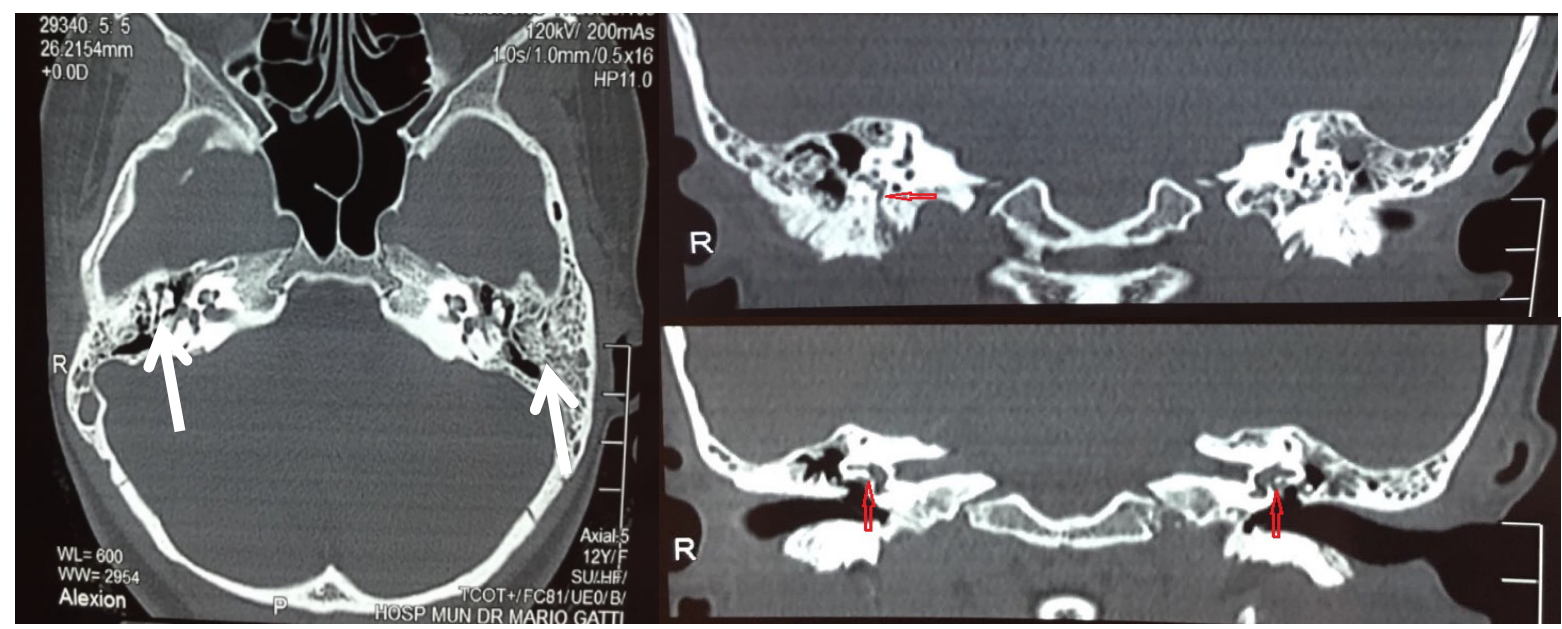

Figure 2. CT scan of temporal bones with bone window. Partial opacification of mastoid air cells (white arrows) and absence of facial nerve canal dehiscence (red arrows)

inside the canal leads to mechanical compression and ischemic neuritis. Persistent inflammation of the middle ear can also cause dehiscence of the facial nerve canal, which can result in facial paralysis [6].

Acute mastoiditis is classically characterized by fever, irritability, otalgia, swelling of the mastoid region, retroauricular erythema. Generally, it is associated with an episode of otitis media 30 days before. However, mastoiditis can occur in a silent or masked manner [7]. Silent mastoiditis occurs when antibiotic therapy provides temporary relief of symptoms, but the inflammation of the middle ear continues silently. Masked mastoiditis refers to mastoiditis without signs or symptoms of acute otitis media. It is related to the clearance of middle ear infection with persistent blockage of the mastoid compartment [8]. Understanding these entities may allow early diagnosis, in addition to promoting the use of radiographic studies [9]. High-resolution CT scan is the imaging modality of choice because it provides better bone details than magnetic resonance imaging. It also allows detecting the extent of deformation of the mastoid air cell, the presence of extradural extension and the integrity of the ossicular chain, the facial nerve canal and the bone labyrinth [8]. In this case report, the radiological diagnosis of mastoiditis was based on CT scan, which revealed opacification of mastoid air cells and absence of facial nerve canal dehiscence (Figure 2).

Eradication of the suppurative process is the most important goal in treating otomastoiditis with facial paralysis [9]. The most common pathogens in mastoiditis are Streptococcus pneumonia, Staphylococcus aureus, Haemophilus influenza and Pseudomonas spp [7]. Treatment should involve a broad-spectrum third generation cephalosporin with meningeal penetration. Initial antibiotic therapy should be adjusted after testing microbiological sensitivity to antibiotics in positive cultures. Due to the low prevalence of facial paralysis, most clinical studies are limited, so it is difficult to determine the ideal treatment. Most authors manage AOM complicated with facial nerve palsy with parenteral antimicrobial therapy and myringotomy. This is due to the frequency of AOM with purulent secretion. In this case report, myringotomy was also performed for this reason. However, some experts advocate a less aggressive approach with tympanic paracentesis and parenteral antibiotics. Many experts believe that the lack of response to myringotomy (or paracentesis) and antibiotics in a few days is an indication of mastoidectomy or, rarely, facial nerve decompression.

The prognosis of otomastoiditis complicated with facial palsy after appropriate therapy is generally excellent. However, one study demonstrated a residual neural dysfunction incidence of $6 \%$ [1]. Therefore, careful investigation of middle ear regions and early treatment must be performed to avoid long-term sequelae ${ }^{1}$. In this case report, we obtained total regression of facial paralysis and clinical symptoms in 20 days, demonstrating the good outcome of the performed treatment.

\section{Conclusion}

Facial nerve paralysis is a rare complication of AOM, especially after the emergence of antibiotic therapy. The causes of facial paralysis due to AOM or otomastoiditis are still poorly understood. $M$ o $s$ authors argue that the gold standard is conservative treatment with antibiotic therapy, corticotherapy and myringotomy.

Mastoidectomy would be reserved for only a few cases, always associated with conservative treatment. As the sequelae have a low incidence rate, facial paralysis due to AOM with or without mastoiditis have an excellent prognosis.

\section{References}

1. Ellefsen B, Bonding P (1996) Facial palsy in acute otitis media. Clin Otolaryngol 21 393-395. [Crossref]

2. Joseph EM, Sperling NM (1998) Facial nerve paralysis in acute otitis media: cause and management revisited. Otolaryngol Head Neck Surg 118: 694-696. [Crossref]

3. Prasad S, Vishwas KV, Pedaprolu S, Kavyashree R (2017) Facial Nerve Paralysis in Acute Suppurative Otitis Media- Management. Indian J Otolaryngol Head Neck Surg 69: 58-61. [Crossref]

4. House JW, Brackmann DE (1985) Facial nerve grading system. Otolaryngol Head Neck Surg 93: 146-147. [Crossref]

5. Chen XC, Lu CW, Liu CH, Wei CC (2014) Facial Palsy Complicated by Masked Otomastoiditis in A 3-Month-Old Infant. J Emerg Med 46: e47-e50. [Crossref]

6. Samuel J, Fernandes CMC (1985) Otogenic complications with an intact tympanic membrane. Laryngoscope 95: 1387-1390. [Crossref]

7. Chien JH, Chen YS, Hung IF, Hsieh KS, Wu KS, et al. (2012) Mastoiditis diagnosed by clinical symptoms and imaging studies in children: disease spectrum and evolving diagnostic challenges. Microbiol Immunol Infect 4: 377-381.

8. Gaio E, Marioni G, de Filippis C, Tregnaghi A, Caltran S, et al. (2004) Facial nerve paralysis secondary to acute otitis media in infants and children. $J$ Paediatr Child Health 40: 483-486. [Crossref]

9. Helms D, Roberge RJ, Kovalick M (2003) Otomastoiditis related facial nerve palsy. $J$ Emerg Med 25: 45-49. [Crossref]

Copyright: (C2020 Teixeira DNA. This is an open-access article distributed under the terms of the Creative Commons Attribution License, which permits unrestricted use, distribution, and reproduction in any medium, provided the original author and source are credited. 